\title{
Disruptive mood dysregulation disorder: current insights
}

This article was published in the following Dove Press journal:

Neuropsychiatric Disease and Treatment

24 August 2016

Number of times this article has been viewed

\section{Raman Baweja \\ Susan D Mayes \\ Usman Hameed \\ James G Waxmonsky}

Department of Psychiatry, Penn

State University College of Medicine,

Hershey, PA, USA
Correspondence: Raman Baweja Department of Psychiatry, Penn State College of Medicine,

500 University Drive, H073,

Hershey, PA 17033-0850, USA

$\mathrm{Tel}+|7| 753|8| 34$

Fax + I 71753 | 649 |

Email rbaweja@hmc.psu.edu
Abstract: Disruptive mood dysregulation disorder (DMDD) was introduced as a new diagnostic entity under the category of depressive disorders in Diagnostic and Statistical Manual of Mental Disorders, Fifth Edition (DSM-5). It was included in DSM-5 primarily to address concerns about the misdiagnosis and consequent overtreatment of bipolar disorder in children and adolescents. DMDD does provide a home for a large percentage of referred children with severe persistent irritability that did not fit well into any DSM, Fourth Edition (DSM-IV) diagnostic category. However, it has been a controversial addition to the DSM-5 due to lack of published validity studies, leading to questions about its validity as a distinct disorder. In this article, the authors discuss the diagnostic criteria, assessment, epidemiology, criticism of the diagnosis, and pathophysiology, as well as treatment and future directions for DMDD. They also review the literature on severe mood dysregulation, as described by the National Institute of Mental Health, as the scientific support for DMDD is based primarily on studies of severe mood dysregulation.

Keywords: disruptive mood dysregulation disorder, persistent irritability, temper outbursts

\section{Introduction}

Since the mid-1990s, there has been sizable debate that mania in children and adolescents presents differently compared to adults. Pediatric onset mania was theorized to present as severe nonepisodic irritability with extended periods of very rapid mood cycling within the day ${ }^{1-3}$ versus discrete mood cycles. ${ }^{4,5}$ With this broader concept of pediatric bipolar disorder in the US, the rate of bipolar disorder diagnosis increased over 40 -fold in less than a decade. ${ }^{6,7}$ The conceptualization of severe nonepisodic irritability as a form of mania has been associated with a sizable increase in the use of mood stabilizers and atypical antipsychotic drugs in children. ${ }^{8}$ This trend is concerning given the potential side effects of these medications and the paucity of long-term safety data in developing children. Substantial controversy ensued about the diagnostic validity of the broad phenotype of pediatric bipolar disorder. ${ }^{9-14}$

\section{Conceptualization of disruptive mood dysregulation disorder}

Regardless of how chronic nonepisodic irritability is categorized diagnostically, there is consensus that it can be severely impairing and merits treatments. ${ }^{10,11,13,15}$ As an alternative to subsuming chronic irritability in children under bipolar disorder, Diagnostic and Statistical Manual of Mental Disorders, Fifth Edition (DSM-5) created the diagnosis, disruptive mood dysregulation disorder (DMDD).

The National Institute of Mental Health (NIMH) proposed a syndrome called "severe mood dysregulation" (SMD) to promote the systematic evaluation of children with 
recurrent temper outbursts and a persistent negative mood. SMD was primarily created to assess if severe nonepisodic irritability belongs to the bipolar spectrum disorder. Validation studies of this syndrome were conducted by comparing it to episodic mania (narrow phenotype of bipolar disorder) on longitudinal course, family history of bipolar disorder, and pathophysiology. ${ }^{13}$ The youth with SMD had extremely high rates $(\geq 75 \%)$ of attention-deficit hyperactivity disorder (ADHD) and oppositional-defiant disorder (ODD), as well as anxiety disorders (58\%). ${ }^{13}$

The primary question addressed in the early initial SMD studies was whether it predicted the development of bipolar disorder. Stringaris et al evaluated the rates of manic episodes in youth with SMD $(\mathrm{N}=84)$ and with bipolar disorder $(\mathrm{N}=93)$ over a follow-up period of approximately 2 years. ${ }^{16}$ Only one youth (1.2\%) with SMD presented with a manic, hypomanic, or mixed episode, as compared to $58(62 \%)$ with bipolar disorder. In a community sample of 776 youth, Leibenluft et al ${ }^{17}$ examined the stability of chronic and episodic irritability at three time points. In this sample, longitudinal stability of irritability was stronger within types than between types. Chronic irritability during early adolescence (mean age 13.8 years) predicted ADHD at late adolescence (mean age 16.2 years) and major depressive disorder in early adulthood (mean age 22.1 years). In comparison, episodic irritability predicted mania. This study concluded that episodic and chronic irritability are distinct constructs. Additional follow-up of the same sample revealed that chronic irritability in adolescence predicted dysthymia, generalized anxiety disorders, and major depressive disorder at 20 years follow-up (mean age 33.2 years). ${ }^{18}$ Other longitudinal follow-up studies also found that youth with DMDD/SMD are at high risk for depressive and anxiety disorders, but not for bipolar disorder. ${ }^{19,20}$ Furthermore, youth with SMD have lower familial rates of bipolar disorder as compared to youth with narrow-phenotype bipolar disorder. ${ }^{21}$ Also, differences between SMD and bipolar disorder have been reported on various pathophysiological markers. ${ }^{13,22-25}$ In contrast, a definition of bipolar disorder not otherwise specified (BP-NOS) that requires the presence of discernable mood cycles in children has been found to be associated with increased risk for the development of full bipolar disorder, suggesting that the presence of episodic irritability is more predictive of bipolar disorder than nonepisodic irritability. ${ }^{26,27}$

\section{Diagnostic criteria for DMDD}

The DMDD criteria are primarily derived from the SMD, albeit with some significant modifications. In the DSM, Fifth Edition (DSM-5), the DMDD diagnosis (Table 1) has two core criteria - severe recurrent temper outbursts and chronic nonepisodic irritability. ${ }^{28}$ Despite its novelty, DMDD is the only diagnosis in the DSM-5 depressive disorder section that requires childhood onset. The DSM-5 specifically states that individuals whose symptoms meet the criteria for both DMDD and ODD should only be given the diagnosis of DMDD. ${ }^{28}$

DMDD differs in several ways from SMD. SMD required recurrent temper outbursts, a persistent negative mood (which, unlike DMDD, includes depressed mood), and the presence of at least three "hyperarousal" symptoms (pressured speech, racing thoughts or flight of ideas, intrusiveness, distractibility, insomnia, and agitation). These hyperarousal criteria were included because it was these symptoms in persistently irritable children that often led to a concern about mania. ${ }^{2,14}$ Also, age of onset for SMD was before age 12 years and the maximum symptom-free period was 2 months.

Table I Diagnostic criteria for DMDD

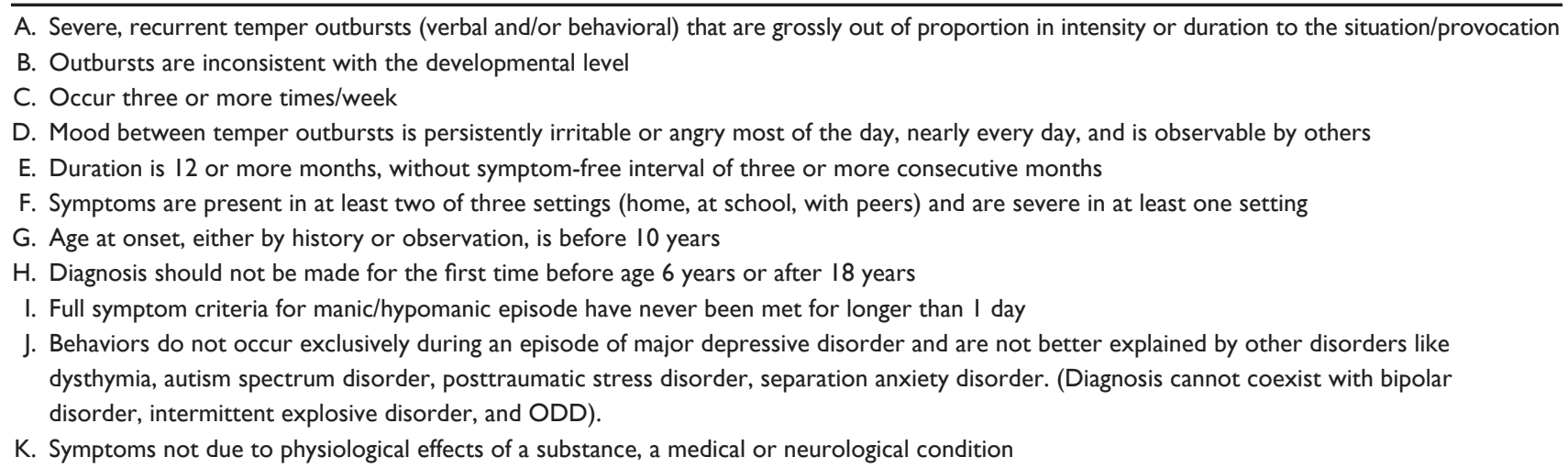

Abbreviations: DMDD, disruptive mood dysregulation disorder; ODD, oppositional-defiant disorder. 


\section{Assessment method for DMDD}

There is no consensus or even well-validated scales for the assessment of DMDD or gold standard measures for the assessment of irritability in children. Most parent and teacher rating scales measuring irritability and tantrums focus on the frequency of such events, with less emphasis on severity. Few measures capture qualitative descriptions of temper outbursts that provide detailed descriptions of the triggers, duration, and intensity of temper outbursts that would be helpful for diagnosing DMDD in children with other oppositional behaviors. There are several established measures for assessing aggressive behaviors, but physical aggression is not a requirement for DMDD as temper outbursts can be verbal and many aggressive youth do not exhibit persistent irritability. Therefore, ratings scales measuring aggression may not be the best assessment tools for DMDD. A sizable percentage of children with temper outbursts and frequent irritable mood will not meet the other criteria for DMDD. ${ }^{29}$ Therefore, it is important to assess all the inclusion and exclusion criteria. In addition, parents may interpret the term "temper outbursts" differently based on the frequency of their child's disruptive behaviors, ${ }^{30}$ so it is important to query parents about details of their child's reaction to frustration or other negative stimuli.

The NIMH research group and others have used versions of the Kiddie Schedule for Affective Disorders and Schizophrenia for School-Aged Children or similar interviews to diagnose DMDD. ${ }^{19,29,31,32}$ None of these interviews have been adapted for widespread clinical use and their administration time is a potential barrier for use in busy clinical services. When evaluating a child in a clinical setting, it is important to assess the triggers for temper outbursts, the duration and frequency of these outbursts, the behaviors commonly exhibited during the outbursts, and what helps to resolve the outbursts, as well as the quality, variability, and severity of the interval mood, the level of impairment caused by the outbursts, and irritability across home, school, and in other social settings. Gathering information from the child and parent, as well as from those adults who work with the child in a setting where they are frequently symptomatic (eg, teachers) is advised. A recent study showed poor agreement between informants on the presence of DMDD symptoms. ${ }^{33}$ Mothers and fathers reported a substantially higher percentage of children with elevated DMDD symptoms (30\% and 25\%), as compared to teachers $(12 \%)$. It seems prudent to obtain ratings from both parents and teachers, which is particularly important for disorders like DMDD that have DSM- 5 crosssetting diagnostic requirements.

\section{Epidemiology}

To date, there have been very few prospective studies on DMDD due to its novelty. However, studies have examined the prevalence of retrospectively diagnosed cases of DMDD or SMD in existing datasets.

In the Great Smoky Mountains Study sample, ${ }^{19,29}$ the lifetime prevalence rates of DMDD (4.4\%) and SMD (3.3\%) were comparable. Copeland et al, ${ }^{29}$ using existing data from three large epidemiological samples including both preschool and school-age cohorts, reported that around half (46\%-49\%) of school-age youth and around $80 \%$ preschoolers were found to have severe temper outbursts in the last 3 months. Among school-aged cohorts, the prevalence dropped to $7 \%$ when the DSM-5 frequency criterion was applied, and it dropped further $(1.5 \%-2.8 \%)$ with the duration criterion. Using the full DSM-5 DMDD criteria, the prevalence rate declined to $~ 1 \%$. In the preschool cohort, the prevalence rate of DMDD using the entire DSM criteria except for age of onset was 3.3\%. The school-age youth with DMDD experienced significant social impairment (relationship with parents, siblings, and teachers), school suspension, and service use (mental health and general medical), reinforcing the findings from other studies that youth with severe nonepisodic irritability are appreciably impaired, even if they do not meet the criteria for bipolar disorder. Similar rates of the core DMDD symptoms were found in another population-based sample of 376 children. ${ }^{34}$ In a large nationally representative sample of adolescents, the prevalence rate of DMDD was $0.12 \%$ using strict criteria for DMDD and increased with relaxation of the mania/hypomania exclusion criterion $(0.56 \%)$, the frequency criterion $(1.71 \%)$, or both $(5.26 \%){ }^{35}$

Higher rates have been reported in clinical samples. Axelson et $\mathrm{a}^{36}$ found that $26 \%$ of children participating in the Longitudinal Assessment of Manic Symptoms (LAMS) study met DMDD criteria. These children were recruited from outpatient clinics and were preselected for the presence of prominent mood lability. Using the same LAMS cohort, Fristad et $\mathrm{al}^{15}$ examined the similarities and differences between DMDD and BP-NOS at baseline and in 3 years. The diagnosis of DMDD was established using operationalized Schedule for Affective Disorders and Schizophrenia for School-Age Children-Present and Lifetime Version criteria from the study by Axelson et al. ${ }^{36}$ The diagnosis of BP-NOS was established using BP-NOS criteria from Course and Outcomes of Bipolar Youth, ${ }^{37}$ which requires the presence of observable mood cycles. In this sample (6-12.9 years of age), chronic irritability was common in both groups. While twice as many 
children were diagnosed with DMDD than with BP-NOS, the groups were similar in most characteristics other than parental history of bipolar disorder and the presence of manic symptoms. In addition, children with DMDD were more likely to be boys and were younger in age. ${ }^{15}$ The comparable severity between groups is a reminder that DMDD merits treatment even if there is no other comorbid psychopathology. Over the 2-year follow-up in LAMS study, $40 \%$ of the sample met DMDD criteria at least once, but $52 \%$ of these participants met the criteria only at one of the three annual assessment points. ${ }^{36}$ In another community sample of 665 children aged 6-12 years, temporal stability was low as well, with $71 \%$ of those with symptoms at baseline no longer having symptoms at follow-up. In addition, only $45 \%$ with DMDD symptoms at follow-up had these symptoms 8 years earlier. ${ }^{38}$

In a large clinical sample $(\mathrm{N}=911)$ of 5-18 year olds referred for problems with behavioral outbursts, SMD was the most common (54.4\%) diagnosis. ${ }^{39}$ In a study of 1,593 children with autism, ADHD, and neurotypical development (6-16 years), mothers reported on the frequency of DMDD symptoms. Percentages of children for whom both irritability and temper outbursts were rated as "often or very often" a problem were $45 \%$ for autism, 39\% for ADHDcombined type, $12 \%$ for ADHD-inattentive type, and 3\% for neurotypical children. ${ }^{40}$ Interestingly, in another study, only $25 \%$ of adolescents with bipolar disorder met the criteria for a lifetime diagnosis of the DMDD phenotype (excluding criterion of onset before age 10), suggesting that persistent irritability and temper outburst are not a common precursor to adolescent mania. ${ }^{41}$

There is limited but expanding literature base on the genetics of DMDD and related constructs such as irritability. ${ }^{42}$ A study comparing parental psychiatric history of young psychiatric referrals with and without DMDD found no differences between groups in parental history of depression, bipolar disorder, anxiety disorder, psychotic disorder, substance use disorder, ADHD, and conduct disorder. ${ }^{36}$ Brotman et al reported that parents of youth who met DSM-IV criteria for bipolar disorder were significantly more likely to be diagnosed with bipolar disorder than parents of youth with SMD, whereas there was no difference in the parental diagnoses of major depressive disorder, anxiety disorder, substance abuse, eating disorder, or schizophrenia between groups. ${ }^{21}$ A study examining rates of DMDD among youth with parents who had bipolar disorder compared to healthy parents and parents with psychiatric disorders other than bipolar disorder found that offspring of parents with bipolar disorder were more likely to have chronic irritably and meet the criteria for DMDD than the children of parents who had other forms of mental illness. ${ }^{43}$

In summary, it appears that DMDD symptoms are relatively common in referred children, but the full disorder is much less common. However, even those with elevated symptoms not meeting full diagnostic criteria experience significant impairment. Rates are substantially higher in clinical samples, especially in those with high rates of externalizing disorders and/or mood lability. However, in many cases, even in clinical samples, the temporal stability of the symptoms is low.

\section{Criticism of DMDD diagnosis}

The development of DMDD has been debated since its inception, in part because it was introduced to the DSM-5 without published validity studies. ${ }^{29,44}$ Moreover, agreement between clinicians on the diagnosis of DMDD was poor in DSM-5 field trials. ${ }^{45}$ Furthermore, the scientific evidence to support DMDD diagnoses derives mainly from studies of SMD, which is related to DMDD, but not identical. ${ }^{13,16,19}$ In addition to nonepisodic irritability, the SMD syndrome also includes sadness and hyperarousal; so, many with SMD would not have DMDD. In the Great Smoky Mountains Study, which assessed overlap between SMD and DMDD, only 38.9\% of youth with SMD met the criteria for DMDD. ${ }^{29}$ Only slightly higher rates of agreement were reported in another community sample, where $58 \%$ of children with SMD met the criteria for DMDD and $47 \%$ of children with DMDD met the criteria for SMD. ${ }^{46}$ The reasons why children with SMD failed to meet the criteria for DMDD were not explained. Nonetheless, these findings question the degree to which results of studies of youth with SMD may be applicable to DMDD.

DMDD and SMD are frequently associated with other psychiatric disorders, most commonly disruptive behavior disorders (especially ODD) and anxiety/mood disorders. ${ }^{13,19,29,36,47}$ In a large nationally representative sample, $92.8 \%$ of DMDD-positive youth met the criteria for another DSM-IV disorder including mood disorders, conduct/ODD, ADHD, and substance abuse. The majority of youth with DMDD had already received treatment for other comorbid disorders, mainly a mood or behavior disorder. ${ }^{35}$ In the LAMS study, $61 \%$ of youth with conduct disorder and $58 \%$ of the participants with ODD met DMDD criteria, while almost all youth with DMDD met the criteria for ODD, making it challenging to differentiate DMDD from ODD. The participants with ODD who met DMDD criteria did not differ significantly from their counterparts without DMDD with respect to symptom severity, comorbidity, or functional 
impairment. ${ }^{36}$ Other studies of clinical samples have reported similar degrees of overlap, ${ }^{38,48}$ suggesting the value of first treating the oppositional behaviors and other externalizing problems. While it is uncommon to find children with just DMDD, when it does it occur in isolation, DMDD is still associated with appreciable impairment. ${ }^{29}$

The concern about the overlap between DMDD and ODD is exacerbated by the DSM-5 preclusion from diagnosing ODD in children meeting the criteria for DMDD. Precluding an ODD diagnosis essentially ignores the impact of argumentative and vindictive behavior, which has been found to make unique contributions to the impairments caused by ODD. ${ }^{49}$ Presently, there is a sizable debate that DMDD may be better served as a modifier of ODD, given that nearly all youth with DMDD meet the criteria for $\mathrm{ODD}^{50}$ and that irritability appears to be a distinct domain from the other symptoms of ODD. ${ }^{49,51}$

\section{Pathophysiology}

The NIMH research group led by Ellen Leibenluft, PhD, has conducted a wide range of studies examining the pathophysiology underlying episodic and nonepisodic irritability utilizing behavioral, neurocognitive, and physiologic measures including functional magnetic resonance imaging (fMRI), eventrelated potentials (ERP), and magnetoencephalography. ${ }^{52}$ However, no study has yet been conducted specifically on children meeting the diagnostic criteria for DMDD.

When frustrated, youth with SMD experience excessive arousal $^{25,47}$ and state-dependent impairments in attentional flexibility. ${ }^{23}$ They exhibit differential patterns of central nervous system (CNS) activation in response to negative feedback, compared to with bipolar disorder, ADHD subjects without irritability, and healthy subjects. ${ }^{52}$ These impairments and patterns of neural activation may contribute to the prolonged recovery from frustration experienced by youth with DMDD that can be particularly impairing for peer relationships. ${ }^{44,53}$ Similar impairments in emotion regulation when under distress have been found in young children with recurrent temper outbursts not formally diagnosed with DMDD. ${ }^{47}$ Youth with SMD also have difficulty identifying negative emotions ${ }^{54}$ and experience greater fear when viewing neutral faces. These impairments in emotion processing have been theorized to cause the elevated rates of reactive aggression seen with SMD. ${ }^{23}$

Impairment in instrumental learning (learning from rewards and consequences) has been theorized to be a core deficit in youth with persistent aggression or irritability. ${ }^{52}$ Studies on youth with SMD report reduced capacity to adapt responses to changing stimuli (reversal learning).
These deficits may be exacerbated when they are frustrated by being blocked from achieving a desirable reward. ${ }^{23,25}$ These behavioral impairments are consistent with fMRI studies of SMD youth showing abnormally reduced activation in neural regions associated with emotional salience, spatial attention, and reward processing in response to frustration tasks. ${ }^{23}$ Some have theorized an enhanced sensitivity to loss may drive the emotional reactivity seen in SMD, as has been observed in anxious youth. ${ }^{13}$ A recent ERP study ${ }^{55}$ showed that DMDD symptoms at age 3 predicted enhanced responsiveness to monetary rewards in preadolescence 6 years later, suggesting that impairment in reward processing may be more salient than just excessive reactivity to loss.

Similar deficits are also seen in youth with bipolar spectrum illness, such as face emotion-labeling deficits, impaired performance on learned response reversal paradigms, and increased subjective distress while performing a frustrating task. ${ }^{13}$ However, the neural circuits mediating these pathophysiologic abnormalities differ between the SMD and bipolar disorder groups. ${ }^{13}$ For example, youth with SMD and bipolar disorder both reported more frustration as compared to healthy controls in a study using a rigged task to elicit frustration. Nevertheless, ERP measures were different between these two groups. During both frustrating and nonfrustrating blocks, youth with SMD had deficits in bottom-up early attentional processes (decreased central, temporal, and parietal N1 and P1 waves), while youth with bipolar disorder had deficient top-down executive attention (decreased parietal P3 waves) specifically during frustration, ${ }^{24}$ most consistent with deficits seen in ADHD. Similarly, on a facial affect recognition task, the participants' level of irritability correlated with amygdala activity across all intensities for all emotions (happy, fearful, and angry faces) in the DMDD group, but only for fearful faces in participants with bipolar disorder. In the ventral visual stream, associations between neural activity and irritability were found more consistently in the DMDD group than in the bipolar disorder group, especially in response to ambiguous angry faces. Authors suggested diagnostic specificity in the neural correlates of irritability and the need to evaluate different approaches to treat irritability in the two disorders. ${ }^{32}$ Differences in functional connectivity between the left basolateral amygdala, the frontal lobes, and the cingulate have also been reported between SMD and bipolar disorder, with hyperconnectivity seen only in youth with bipolar disorder. ${ }^{56}$ On magnetoencephalography, youth with SMD displayed significantly greater activation of the medial frontal gyrus and the anterior cingulate cortex in 
response to negative feedback, whereas youth with bipolar disorder had decreased insula activation and greater superior frontal gyrus activation. ${ }^{25}$

\section{Treatment}

Conceptualizing severe, nonepisodic irritability as a broad phenotype of bipolar disorder may lead to reluctance to treat youth with DMDD with CNS stimulants or antidepressants because of the risks for worsening irritability and precipitating mania. However, if youth with these conditions are more similar in pathophysiology to children with depression, ADHD, and anxiety disorders, the risks for mania is low. Therefore, selective serotonin reuptake inhibitors and CNS stimulants would be reasonable options. Given the appreciable short- and long-term side effects of atypical antipsychotics and other antimanic medications compared to medications for depression, anxiety, or ADHD, ${ }^{57}$ this differentiation is important. Studies examining the course and pathophysiology of DMDD support this latter formulation, as more commonalties have been found with ODD, ADHD, anxiety, and depression than with bipolar disorder. Preliminary evidence suggests that youth with ADHD and DMDD or with $\mathrm{ADHD}$ and recurrent aggression respond positively to CNS stimulants, with little risk of manic activation or adverse emotional effects. ${ }^{58-62}$ Similarly, psychosocial techniques derived from those employed for ADHD, ODD, and depression have been found to be efficacious. ${ }^{31,63}$ Current studies have not found any evidence of increased risks of these treatments beyond those seen in youth with just a behavioral disorder. However, existing treatments for other disorders do not appear sufficient to optimize functioning. ${ }^{31,59,64}$ Therefore, it is imperative to develop novel treatments tailored to address the specific deficits associated with DMDD.

As irritability is associated with a wide range of disorders and is a common reaction to negative life events in children, it is imperative for clinicians to explore all the potential causes of chronic irritability rather than ending the inquiry once a diagnosis of DMDD is reached. These include examination for conflicts within the family, at school, or in other settings, as well as for evidence of past trauma and a wide range of psychiatric disorders. Meeting the criteria for DMDD should not stop the search for triggers for the child's irritability, as DMDD does not identify an etiology for the child's distress. In fact, any efficacious psychosocial treatment for DMDD will likely necessitate some degree of antecedent management, ${ }^{31}$ making it even more important to identify environmental stressors. This approach is more likely to facilitate a personalized treatment that incorporates psychosocial interventions, liaison with the school, and involvement of all available community resources to treat the actual functional impairments versus sole reliance on medication in an attempt to reduce irritability or aggression. An individually tailored, multiprong approach is most likely to optimize treatment response.

No formal treatment studies of youth with DMDD have been conducted. However, there is an expanding database for SMD and related conditions (eg, ADHD plus aggression, ADHD, and ODD). While SMD is the most similar diagnostic construct to DMDD, it is important to emphasize that it is not presently clear how well treatment effects for SMD translate to DMDD.

\section{Pharmacological intervention}

The only randomized, placebo-controlled trial of medication in children with SMD found no benefit of lithium over placebo. ${ }^{65}$ However, others have examined medication effects in related phenotypes. A controlled trial in youth $(\mathrm{N}=27)$ with ADHD and aggressive behavior refractory to stimulants monotherapy (a phenotype similar to SMD) found divalproex sodium combined with CNS stimulants and behavioral therapy to be more effective than placebo combined with a CNS stimulant and behavioral therapy. ${ }^{66}$ In both these studies, all participants also received psychosocial treatments prior to the randomization. In Blader et al's study, CNS stimulant dose was optimized prior to assignment to divalproex sodium or placebo. Approximately half of the samples in each study improved to the degree that they no longer met the entry criteria. ${ }^{66}$

Behavioral and medication treatments targeting ADHD symptoms in the Multimodal Treatment Study of Children with ADHD were associated with reduced levels of irritability in children with ADHD. Furthermore, irritability did not appear to influence the treatment response for ADHD symptoms. ${ }^{67}$ Waxmonsky et al also observed that CNS stimulants were equally efficacious and were tolerated in ADHD youth with and without SMD symptoms. ${ }^{60}$ In a prospective open-label trial of stimulants in youth with ADHD who also met the full diagnostic criteria for SMD, both methylphenidate and amphetamine preparations were well tolerated by children, and were associated with clinically significant reduction in externalizing symptoms but only mild improvements in mood symptoms. Moreover, most participants still exhibited significant global impairment after the CNS stimulant was optimized. ${ }^{64}$ These combined results suggest the value of first optimizing treatment for ADHD and other comorbid conditions prior to pharmacologically targeting irritability or aggression. 
There are growing concerns about the increasingly common trends of using the combination of CNS stimulants and antipsychotics to treat persistent aggression and other behavioral problems. ${ }^{68}$ The majority of antipsychotic prescriptions in children are for off-label indications, such as the treatment of aggression in ADHD. ${ }^{69}$ However, we are only beginning to systematically evaluate the efficacy of this practice. An open-label trial of risperidone (mean dose $=1.28 \mathrm{mg}$ ) in youth with SMD showed significant reduction in irritability scores..$^{70}$ In a randomized, controlled trial, augmenting psychostimulant and parent training with risperidone versus placebo in children with ADHD and severe aggression led to moderate but variable improvement in aggressive behaviors over 9 weeks. Prolactin elevation and gastrointestinal upset were more with risperidone, while weight gain was minor (The Treatment of Severe Childhood Aggression study). ${ }^{62}$ Augmentation with risperidone was also superior to placebo in reduction of severity of ADHD and ODD symptoms and associated impairment; however, clinical improvement was context specific and effect sizes ranged from small to moderate. ${ }^{71}$ At the end of 52 weeks, around $23 \%$ of youth with risperidone augmentation stopped taking medications versus $11 \%$ on placebo. Risperidone augmentation was associated with sustained elevated prolactin levels (mean level $21.56 \mathrm{ng} / \mathrm{mL}$ ), and there was a decrease in weight over time only in the placebo arm. ${ }^{72}$ These results suggest that antipsychotic medications may be most appropriate for children with DMDD who are in need of a rapid reduction in aggressive behaviors after optimizing treatment of their ADHD.

\section{Psychotherapeutic intervention}

Evidence-based parent training interventions and other psychotherapeutic interventions should always be considered in the treatment of DMDD, given the high degree of overlap with ODD and the established efficacy of these treatments for reducing oppositional behaviors. ${ }^{73}$ Treatment algorithms for aggression in children emphasize the role of these modalities prior to engaging in polypharmacy. ${ }^{74,75} \mathrm{In}$ a randomized controlled trial of 112 children (mean age 5.2 years) with ODD symptoms, parental intervention was more effective in emotional-dysregulated youth than in those with just headstrong characteristics. ${ }^{76}$ Waxmonsky et al found behavioral modification interventions were equally effective in ADHD youth with and without SMD symptoms. ${ }^{60}$ However, those with SMD remained appreciably more impaired than those with ADHD/ODD, suggesting the need for behavioral interventions tailored to the specific deficits and impairments seen within SMD/DMDD. Waxmonsky et al conducted a randomized trial of an integrative group therapy for children with SMD and ADHD. ${ }^{31}$ The parenting intervention was derived from evidence-based treatments for pediatric behavioral problems with an emphasis on managing antecedents for temper outbursts and training parents to be effective emotion regulation coaches for their children. The children's group primarily addressed impairments in affect identification, soothing of negative affective states, and problem solving, with an emphasis on soothing negative mood states prior to problem solving. The joint treatment package was found to be feasible and well received by families, with greater reduction in parent-rated irritability than with CNS stimulants alone. In those completing the majority of sessions, additional improvement in mood symptoms were seen. ${ }^{31}$

\section{Future directions}

It has now been established that severe nonepisodic irritability is not a meaningful risk factor for mania. However, the potential impact of irritability is still substantial, given its prevalence and appreciable morbidity in youth with a wide range of psychopathology. ${ }^{77}$ Therefore, the field needs to expand efforts to systematically assess the complex construct of irritability. Future work should focus on identifying the underlying neurophysiological profiles associated with severe, persistent irritability as well as understanding the mechanistic pathways driving it, in order to develop improved assessment measures and more efficacious treatments.

Initial data on the safety and efficacy of CNS stimulants are encouraging, but randomized controlled trials in youth meeting the full diagnostic criteria for DMDD are needed. Given the high rates of comorbid anxiety in youth with DMDD, the efficacy and tolerability of effective anxiolytic treatment in children should also be explored. Currently, the NIMH is conducting a trial examining the effects of citalopram. ${ }^{78}$ Likewise, psychosocial interventions efficacious for ODD and anxiety disorders should also be studied under randomized control group conditions over an extended timeframe.

\section{Summary}

In DSM-5, the novel diagnosis of DMDD was added to address concerns about the misdiagnosis and consequent overtreatment of bipolar disorder in children and adolescents. DMDD does provide a home for a large percentage of referred children with severe persistent irritability that did not fit well into any DSM-IV diagnostic category. However, DMDD symptoms are found in many psychiatric disorders and rarely occur in isolation, to the degree that the 
formulation of DMDD as a unique and separate disorder is not well supported by the present literature base. Therefore, an alternative proposal of viewing DMDD as a modifier of ODD has been proposed. Regardless of where one stands on this diagnostic debate, it is clear that youth who have persistent, explosive irritability and recurrent temper outbursts are highly impaired and in need of evidence-based treatments. Further research on this topic is needed to guide the development of these treatments.

\section{Disclosure}

In the past 2 years, Dr JG Waxmonsky has received research funding from the National Institutes of Health, Noven Pharmaceuticals, and Shire; served on the advisory boards of Noven and Iron Shore; and provided Continuing Medical Education talks funded by Quintiles.

Dr R Baweja, Dr SD Mayes, and Dr U Hameed have no conflicts of interest to report in this work.

\section{References}

1. Biederman J, Klein RG, Pine DS, Klein DF. Resolved: mania is mistaken for ADHD in prepubertal children. J Am Acad Child Adolesc Psychiatry. 1998;37(10):1091-1096, discussion 1096-1099.

2. Faraone SV, Biederman J, Mennin D, Wozniak J, Spencer T. Attentiondeficit hyperactivity disorder with bipolar disorder: a familial subtype? J Am Acad Child Adolesc Psychiatry. 1997;36(10):1378-1387, discussion $1387-1390$

3. Wozniak J, Biederman J, Kiely K, et al. Mania-like symptoms suggestive of childhood-onset bipolar disorder in clinically referred children. J Am Acad Child Adolesc Psychiatry. 1995;34(7):867-876.

4. American Psychiatric Association. Diagnostic and Statistical Manual of Mental Disorders. 4th ed. Washington, DC: American Psychiatric Press; 1994.

5. Geller B, Williams M, Zimerman B, Frazier J, Beringer L, Warner KL. Prepubertal and early adolescent bipolarity differentiate from ADHD by manic symptoms, grandiose delusions, ultra-rapid or ultradian cycling. $J$ Affect Disord. 1998;51(2):81-91.

6. Blader JC, Carlson GA. Increased rates of bipolar disorder diagnoses among U.S. child, adolescent, and adult inpatients, 1996-2004. Biol Psychiatry. 2007;62(2):107-114.

7. Moreno C, Laje G, Blanco C, Jiang H, Schmidt AB, Olfson M. National trends in the outpatient diagnosis and treatment of bipolar disorder in youth. Arch Gen Psychiatry. 2007;64(9):1032-1039.

8. Correll CU, Blader JC. Antipsychotic use in youth without psychosis: a double-edged sword. JAMA Psychiatry. 2015;72(9):859-860.

9. Althoff RR. Dysregulated children reconsidered. J Am Acad Child Adolesc Psychiatry. 2010;49(4):302-305.

10. Biederman J, Faraone SV, Wozniak J, Mick E, Kwon A, Aleardi M. Further evidence of unique developmental phenotypic correlates of pediatric bipolar disorder: findings from a large sample of clinically referred preadolescent children assessed over the last 7 years. $J$ Affect Disord. 2004;82(Suppl 1):S45-S58.

11. Carlson GA, Glovinsky I. The concept of bipolar disorder in children: a history of the bipolar controversy. Child Adolesc Psychiatr Clin N Am. 2009;18(2):257-271, vii.

12. Diler RS, Birmaher B, Axelson D, et al. The Child Behavior Checklist (CBCL) and the CBCL-bipolar phenotype are not useful in diagnosing pediatric bipolar disorder. J Child Adolesc Psychopharmacol. 2009;19(1):23-30.
13. Leibenluft E. Severe mood dysregulation, irritability, and the diagnostic boundaries of bipolar disorder in youths. Am J Psychiatry. 2011; 168(2):129-142.

14. Mick E, Spencer T, Wozniak J, Biederman J. Heterogeneity of irritability in attention-deficit/hyperactivity disorder subjects with and without mood disorders. Biol Psychiatry. 2005;58(7):576-582.

15. Fristad MA, Wolfson H, Algorta GP, et al. Disruptive mood dysregulation disorder and bipolar disorder not otherwise specified: fraternal or identical twins? J Child Adolesc Psychopharmacol. 2016;26(2): 138-146.

16. Stringaris A, Baroni A, Haimm C, et al. Pediatric bipolar disorder versus severe mood dysregulation: risk for manic episodes on follow-up. $J \mathrm{Am}$ Acad Child Adolesc Psychiatry. 2010;49(4):397-405.

17. Leibenluft E, Cohen P, Gorrindo T, Brook JS, Pine DS. Chronic versus episodic irritability in youth: a community-based, longitudinal study of clinical and diagnostic associations. J Child Adolesc Psychopharmacol. 2006;16(4):456-466.

18. Stringaris A, Cohen P, Pine DS, Leibenluft E. Adult outcomes of youth irritability: a 20-year prospective community-based study. Am J Psychiatry. 2009;166(9):1048-1054.

19. Brotman MA, Schmajuk M, Rich BA, et al. Prevalence, clinical correlates, and longitudinal course of severe mood dysregulation in children. Biol Psychiatry. 2006;60(9):991-997.

20. Copeland WE, Shanahan L, Egger H, Angold A, Costello EJ. Adult diagnostic and functional outcomes of DSM-5 disruptive mood dysregulation disorder. Am J Psychiatry. 2014;171(6):668-674.

21. Brotman MA, Kassem L, Reising MM, et al. Parental diagnoses in youth with narrow phenotype bipolar disorder or severe mood dysregulation. Am J Psychiatry. 2007;164(8):1238-1241.

22. Brotman MA, Rich BA, Guyer AE, et al. Amygdala activation during emotion processing of neutral faces in children with severe mood dysregulation versus ADHD or bipolar disorder. Am J Psychiatry. 2010;167(1): 61-69.

23. Deveney CM, Connolly ME, Haring CT, et al. Neural mechanisms of frustration in chronically irritable children. Am J Psychiatry. 2013;170(10): 1186-1194.

24. Rich BA, Schmajuk M, Perez-Edgar KE, Fox NA, Pine DS, Leibenluft E. Different psychophysiological and behavioral responses elicited by frustration in pediatric bipolar disorder and severe mood dysregulation. Am J Psychiatry. 2007;164(2):309-317.

25. Rich BA, Carver FW, Holroyd T, et al. Different neural pathways to negative affect in youth with pediatric bipolar disorder and severe mood dysregulation. J Psychiatr Res. 2011;45(10):1283-1294.

26. Birmaher B, Axelson D, Strober M, et al. Clinical course of children and adolescents with bipolar spectrum disorders. Arch Gen Psychiatry. 2006;63(2):175-183.

27. Towbin K, Axelson D, Leibenluft E, Birmaher B. Differentiating bipolar disorder-not otherwise specified and severe mood dysregulation. $J \mathrm{Am}$ Acad Child Adolesc Psychiatry. 2013;52(5):466-481.

28. American Psychiatric Association. Diagnostic and Statistical Manual of Mental Disorders. 5th ed. Washington, DC: American Psychiatric Press; 2013.

29. Copeland WE, Angold A, Costello EJ, Egger H. Prevalence, comorbidity, and correlates of DSM-5 proposed disruptive mood dysregulation disorder. Am J Psychiatry. 2013;170(2):173-179.

30. Carlson GA, Danzig AP, Dougherty LR, Bufferd SJ, Klein DN. Loss of temper and irritability: the relationship to tantrums in a community and clinical sample. J Child Adolesc Psychopharmacol. 2016;26(2):114-122.

31. Waxmonsky JG, Waschbusch DA, Belin P, et al. A randomized clinical trial of an integrative group therapy for children with severe mood dysregulation. J Am Acad Child Adolesc Psychiatry. 2016;55(3):196-207.

32. Wiggins JL, Brotman MA, Adleman NE, et al. Neural correlates of irritability in disruptive mood dysregulation and bipolar disorders. Am J Psychiatry. 2016;173(7):722-730.

33. Mayes SD, Waxmonsky JD, Waschbusch DA, et al. Mother, father, and teacher agreement on disruptive mood dysregulation symptoms in a child psychiatric sample. Int J Ment Health Psychiatry. 2016;2(2). 
34. Mayes SD, Waxmonsky JD, Calhoun SL, Bixler EO. Disruptive mood dysregulation disorder symptoms and association with oppositional defiant and other disorders in a general population child sample. J Child Adolesc Psychopharmacol. 2016;26(2):101-106.

35. Althoff RR, Crehan ET, He JP, Burstein M, Hudziak JJ, Merikangas KR. Disruptive mood dysregulation disorder at ages 13-18: results from the national comorbidity survey-adolescent supplement. $J$ Child Adolesc Psychopharmacol. 2016;26(2):107-113.

36. Axelson D, Findling RL, Fristad MA, et al. Examining the proposed disruptive mood dysregulation disorder diagnosis in children in the Longitudinal Assessment of Manic Symptoms study. J Clin Psychiatry. 2012;73(10):1342-1350

37. Birmaher B, Axelson D, Goldstein B, et al. Four-year longitudinal course of children and adolescents with bipolar spectrum disorders: the Course and Outcome of Bipolar Youth (COBY) study. Am J Psychiatry. 2009;166(7):795-804.

38. Mayes SD, Mathiowetz C, Kokotovich C, et al. Stability of disruptive mood dysregulation disorder symptoms (Irritable-Angry Mood and Temper Outbursts) throughout childhood and adolescence in a general population sample. J Abnorm Child Psychol. 2015;43(8): 1543-1549.

39. Carlson GA, Dyson M. Diagnostic implications of informant disagreement about rage outbursts: bipolar disorder or another condition? Isr J Psychiatry Relat Sci. 2012;49(1):44-51.

40. Mayes SD, Waxmonsky J, Calhoun SL, Kokotovich C, Mathiowetz C, Baweja R. Disruptive mood dysregulation disorder (DMDD) symptoms in children with autism, ADHD, and neurotypical development and impact of co-occurring ODD, depression, and anxiety. Res Autism Spectr Disord. 2015;18:64-72.

41. Mitchell RH, Timmins V, Collins J, Scavone A, Iskric A, Goldstein BI. Prevalence and correlates of disruptive mood dysregulation disorder among adolescents ith bipolar disorder. J Child Adolesc Psychopharmacol. 2016;26(2):147-153.

42. Vidal-Ribas P, Brotman MA, Valdivieso I, Leibenluft E, Stringaris A. The status of irritability in psychiatry: a conceptual and quantitative review. J Am Acad Child Adolesc Psychiatry. 2016;55(7):556-570.

43. Sparks GM, Axelson DA, Yu H, et al. Disruptive mood dysregulation disorder and chronic irritability in youth at familial risk for bipolar disorder. J Am Acad Child Adolesc Psychiatry. 2014;53(4): 408-416.

44. Roy AK, Lopes V, Klein RG. Disruptive mood dysregulation disorder: a new diagnostic approach to chronic irritability in youth. $\mathrm{Am} J$ Psychiatry. 2014;171(9):918-924.

45. Regier DA, Narrow WE, Clarke DE, et al. DSM-5 field trials in the United States and Canada, Part II: test-retest reliability of selected categorical diagnoses. Am J Psychiatry. 2013;170(1):59-70.

46. Dougherty LR, Smith VC, Bufferd SJ, et al. DSM-5 disruptive mood dysregulation disorder: correlates and predictors in young children. Psychol Med. 2014;44(11):2339-2350.

47. Roy AK, Klein RG, Angelosante A, et al. Clinical features of young children referred for impairing temper outbursts. $J$ Child Adolesc Psychopharmacol. 2013;23(9):588-596.

48. Freeman AJ, Youngstrom EA, Youngstrom JK, Findling RL. Disruptive mood dysregulation disorder in a community mental health clinic: prevalence, comorbidity and correlates. J Child Adolesc Psychopharmacol. 2016;26(2):123-130.

49. Burke JD, Hipwell AE, Loeber R. Dimensions of oppositional defiant disorder as predictors of depression and conduct disorder in preadolescent girls. J Am Acad Child Adolesc Psychiatry. 2010;49(5):484-492.

50. Lochman JE, Evans SC, Burke JD, et al. An empirically based alternative to DSM-5's disruptive mood dysregulation disorder for ICD-11. World Psychiatry. 2015;14(1):30-33.

51. Stringaris A, Goodman R. Longitudinal outcome of youth oppositionality: irritable, headstrong, and hurtful behaviors have distinctive predictions. J Am Acad Child Adolesc Psychiatry. 2009;48(4):404-412.

52. Leibenluft E, Stoddard J. The developmental psychopathology of irritability. Dev Psychopathol. 2013;25(4 Pt 2):1473-1487.
53. Bunford N, Evans SW, Becker SP, Langberg JM. Attention-deficit/ hyperactivity disorder and social skills in youth: a moderated mediation model of emotion dysregulation and depression. J Abnorm Child Psychol. 2015;43(2):283-296.

54. Guyer AE, McClure EB, Adler AD, et al. Specificity of facial expression labeling deficits in childhood psychopathology. J Child Psychol Psychiatry. 2007;48(9):863-871.

55. Kessel EM, Dougherty LR, Kujawa A, Hajcak G, Carlson GA, Klein DN. Longitudinal associations between preschool disruptive mood dysregulation disorder symptoms and neural reactivity to monetary reward during preadolescence. J Child Adolesc Psychopharmacol. 2016;26(2): 131-137.

56. Stoddard J, Hsu D, Reynolds RC, et al. Aberrant amygdala intrinsic functional connectivity distinguishes youths with bipolar disorder from those with severe mood dysregulation. Psychiatry Res. 2015;231(2): $120-125$.

57. Correll CU, Manu P, Olshanskiy V, Napolitano B, Kane JM, Malhotra AK. Cardiometabolic risk of second-generation antipsychotic medications during first-time use in children and adolescents. JAMA. 2009;302(16): $1765-1773$.

58. Carlson GA, Loney J, Salisbury H, Kramer JR, Arthur C. Stimulant treatment in young boys with symptoms suggesting childhood mania: a report from a longitudinal study. $J$ Child Adolesc Psychopharmacol. 2000;10(3):175-184.

59. Galanter CA, Carlson GA, Jensen PS, et al. Response to methylphenidate in children with attention deficit hyperactivity disorder and manic symptoms in the multimodal treatment study of children with attention deficit hyperactivity disorder titration trial. $J$ Child Adolesc Psychopharmacol. 2003;13(2):123-136.

60. Waxmonsky J, Pelham WE, Gnagy E, et al. The efficacy and tolerability of methylphenidate and behavior modification in children with attention-deficit/hyperactivity disorder and severe mood dysregulation. J Child Adolesc Psychopharmacol. 2008;18(6):573-588.

61. Blader JC, Pliszka SR, Jensen PS, Schooler NR, Kafantaris V. Stimulantresponsive and stimulant-refractory aggressive behavior among children with ADHD. Pediatrics. 2010;126(4):e796-e806.

62. Aman MG, Bukstein OG, Gadow KD, et al. What does risperidone add to parent training and stimulant for severe aggression in child attentiondeficit/hyperactivity disorder? J Am Acad Child Adolesc Psychiatry. 2014;53(1):47-60.e41.

63. Waxmonsky JG, Wymbs FA, Pariseau ME, et al. A novel group therapy for children with ADHD and severe mood dysregulation. J Atten Disord. 2013;17(6):527-541.

64. Baweja R, Belin PJ, Humphrey HH, et al. The effectiveness and tolerability of central nervous system stimulants in school-age children with attention-deficit/hyperactivity disorder and disruptive mood dysregulation disorder across home and school. J Child Adolesc Psychopharmacol. 2016;26(2):154-163.

65. Dickstein DP, Towbin KE, Van Der Veen JW, et al. Randomized double-blind placebo-controlled trial of lithium in youths with severe mood dysregulation. J Child Adolesc Psychopharmacol. 2009;19(1): 61-73.

66. Blader JC, Schooler NR, Jensen PS, Pliszka SR, Kafantaris V. Adjunctive divalproex versus placebo for children with ADHD and aggression refractory to stimulant monotherapy. Am J Psychiatry. 2009; 166(12):1392-1401.

67. Fernandez de la Cruz L, Simonoff E, McGough JJ, Halperin JM, Arnold LE, Stringaris A. Treatment of children with attention-deficit/ hyperactivity disorder (ADHD) and irritability: results from the multimodal treatment study of children with ADHD (MTA). J Am Acad Child Adolesc Psychiatry. 2015;54(1):62-70.e63.

68. Carlson GA. The dramatic rise in neuroleptic use in children: why do we do it and what does it buy us? Theories from inpatient data 1988-2010. J Child Adolesc Psychopharmacol. 2013;23(3):144-147.

69. Olfson M, King M, Schoenbaum M. Treatment of young people with antipsychotic medications in the United States. JAMA Psychiatry. 2015;72(9):867-874. 
70. Krieger FV, Pheula GF, Coelho R, et al. An open-label trial of risperidone in children and adolescents with severe mood dysregulation. J Child Adolesc Psychopharmacol. 2011;21(3):237-243.

71. Gadow KD, Arnold LE, Molina BS, et al. Risperidone added to parent training and stimulant medication: effects on attention-deficit/ hyperactivity disorder, oppositional defiant disorder, conduct disorder, and peer aggression. J Am Acad Child Adolesc Psychiatry. 2014;53(9):948-959.e941.

72. Gadow KD, Brown NV, Arnold LE, et al. Severely aggressive children receiving stimulant medication versus stimulant and risperidone: 12-month follow-up of the TOSCA trial. J Am Acad Child Adolesc Psychiatry. 2016;55(6):469-478.

73. Fabiano GA, Pelham WE Jr, Coles EK, Gnagy EM, Chronis-Tuscano A, O'Connor BC. A meta-analysis of behavioral treatments for attentiondeficit/hyperactivity disorder. Clin Psychol Rev. 2009;29(2): 129-140.

74. Jensen PS, Arnold LE, Swanson JM, et al. 3-year follow-up of the NIMH MTA study. J Am Acad Child Adolesc Psychiatry. 2007;46(8): 989-1002.
75. Pappadopulos E, Macintyre Ii JC, Crismon ML, et al. Treatment recommendations for the use of antipsychotics for aggressive youth (TRAAY). Part II. J Am Acad Child Adolesc Psychiatry. 2003;42(2):145-161.

76. Scott S, O'Connor TG. An experimental test of differential susceptibility to parenting among emotionally-dysregulated children in a randomized controlled trial for oppositional behavior. J Child Psychol Psychiatry. 2012; 53(11):1184-1193.

77. Hameed U, Dellasega C. Irritability in pediatric patients: normal or not? Prim Care Companion CNS Disord. 2016;18(2).

78. Leibenluft E. National Institute of Mental Health (NIMH). A Controlled Trial of Serotonin Reuptake Inhibitors Added to Stimulant Medication in Youth With Severe Mood Dysregulation. ClinicalTrials. gov [Internet]. Bethesda, MD: National Library of Medicine (US); 2000 [cited May 31, 2016]. Available from: https://clinicaltrials.gov/ct/show/ NCT00794040. Accessed June 8, 2016.
Neuropsychiatric Disease and Treatment

\section{Publish your work in this journal}

Neuropsychiatric Disease and Treatment is an international, peerreviewed journal of clinical therapeutics and pharmacology focusing on concise rapid reporting of clinical or pre-clinical studies on a range of neuropsychiatric and neurological disorders. This journal is indexed on PubMed Central, the 'PsycINFO' database and CAS,

\section{Dovepress}

and is the official journal of The International Neuropsychiatric Association (INA). The manuscript management system is completely online and includes a very quick and fair peer-review system, which is all easy to use. Visit http://www.dovepress.com/testimonials.php to read real quotes from published authors. 